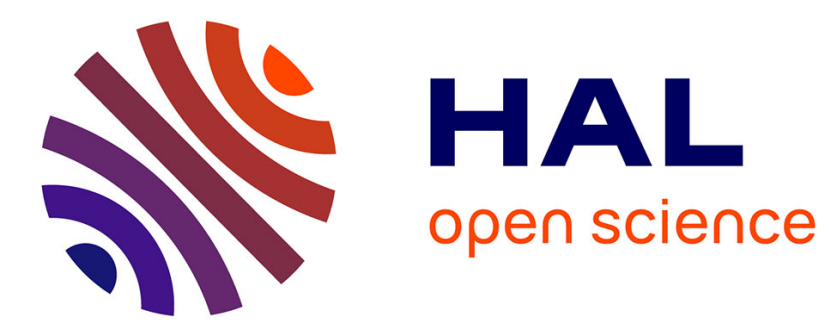

\title{
Étude de bruit neutronique d'un réacteur à eau bouillante par une méthode stochastique
}

\author{
M. Matthey
}

\section{To cite this version:}

M. Matthey. Étude de bruit neutronique d'un réacteur à eau bouillante par une méthode stochastique. Revue de Physique Appliquée, 1976, 11 (4), pp.489-496. 10.1051/rphysap:01976001104048900 . jpa00244084

\section{HAL Id: jpa-00244084 https://hal.science/jpa-00244084}

Submitted on 1 Jan 1976

HAL is a multi-disciplinary open access archive for the deposit and dissemination of scientific research documents, whether they are published or not. The documents may come from teaching and research institutions in France or abroad, or from public or private research centers.
L'archive ouverte pluridisciplinaire HAL, est destinée au dépôt et à la diffusion de documents scientifiques de niveau recherche, publiés ou non, émanant des établissements d'enseignement et de recherche français ou étrangers, des laboratoires publics ou privés. 


\author{
Classification \\ Physics Abstracts \\ $1.650-4.320-4.610$

\section{ÉTUDE DE BRUIT NEUTRONIQUE D'UN RÉACTEUR A EAU BOUILlante PAR UNE MÉTHODE STOCHASTIQUE}

\author{
M. MATTHEY \\ Laboratoire de Génie Atomique de l'Ecole Polytechnique Fédérale de Lausanne, Suisse
}

(Reçu le 22 décembre 1975, accepté le 16 mars 1976)

\begin{abstract}
Résumé. - Un formalisme stochastique basé sur l'équation de Chapman-Kolmogorov permet de décrire le comportement d'un réacteur à eau bouillante, considéré comme ponctuel et soumis à un processus de Markov. La densité spectrale énergétique du bruit neutronique (DSN), la fonction de transfert (FT) et le bruit d'entrée du réacteur sont calculés et une forme analytique approchée est proposée pour chacune de ces fonctions. Une étude paramétrique en fonction de la puissance du réacteur et du coefficient de contre-réaction de vide est présentée ainsi que l'interprétation de l'existence d'une résonance sur la DSN et la FT. Les limites de validité des approximations sont indiquées.
\end{abstract}

Abstract. - A stochastic formalism based on the Chapman-Kolmogorov equation allows us to describe the behaviour of a boiling water reactor, considered as punctual and subject to a Markov process. Neutron power spectral density (DSN), transfer function (FT) and input noise are calculated and an approximate analytical expression is proposed for each function. A parametric study, with reactor power and void coefficient as variables, is performed and an interpretation for the presence of a peak on the DSN and FT is also proposed. The validity limits of the approximations are given.

1. Introduction et hypothèses générales. - Le caractère aléatoire des événements affectant la population neutronique d'un réacteur nucléaire a conduit de nombreux auteurs à considérer ce dernier comme un système physique décrit par un processus de Markov. Ce type d'approche a été particulièrement utilisé pour la description de réacteurs de puissance nulle et de nombreuses expériences ont permis d'en vérifier la validité (cf. par exemple [1]). D. R. Harris [2] et P. R. Pluta [3] ont été parmi les premiers à utiliser les méthodes stochastiques pour décrire le comportement et les caractéristiques des réacteurs de puissance, dans lesquels les effets de contre-réaction jouent un rôle primordial sur les fluctuations internes de réactivité. Diverses sources de bruit des réacteurs de puissance (fluctuations de la température du combustible et du réfrigérant, fluctuations du débit massique du réfrigérant) ont été étudiées par K. Saito [4] et N. Morishima [5] en utilisant un modèle ponctuel du réacteur. Nous développons dans cette étude un modèle théorique simple d'un réacteur à eau bouillante, considéré comme ponctuel avec un seul groupe de neutrons retardés. Nous admettons de plus que les fluctuations des caractéristiques physiques du réfrigérant, telles sa température, son débit massique ou sa pression, sont négligeables. Le réfrigérant constitue un réservoir infini de chaleur à température constante. Nous négligeons également l'effet dû aux mouvements aléatoires des bulles dans le réfrigérant ; ces différentes simplifications permettent une description analytique de la source de bruit globale due aux effets de contreréaction de vide. Signalons que récemment $\mathrm{G}$. Kosaly et al. [6] ont proposé un modèle spatial simple tenant compte de l'effet des sources locales de bruit (négligées ici) sur les fluctuations de la population neutronique d'un réacteur à eau bouillante.

2. Le modèle. - Le traitement théorique des équations stochastiques est semblable à celui exposé dans les articles de N. Morishima [5] et M. Lax [7]. Pour la formulation des effets de contre-réaction, il est fait référence aux publications de R. P. Pluta [3] et R. Isnard [8].

En utilisant la théorie des processus aléatoires [9], nous pouvons écrire l'équation de Chapman-Kolmogorov suivante :

$$
\begin{aligned}
& P\left(\mathbf{y}, t+\Delta t \mid \mathbf{y}_{0}, t_{0}\right)= \\
& \quad=\sum_{\mathbf{y}^{\prime}} P\left(\mathbf{y}^{\prime}, t \mid \mathbf{y}_{0}, t_{0}\right) . P\left(\mathbf{y}^{\prime} \rightarrow \mathbf{y}, \Delta t\right)
\end{aligned}
$$

avec :

$$
P\left(\mathbf{y}, t \mid \mathbf{y}_{0}, t_{0}\right)
$$

probabilité conditionnelle d'existence de l'état $\mathbf{y}$ au temps $t$, étant donné l'état $\mathbf{y}_{0}$ au temps $t_{0}$;

$$
P\left(\mathbf{y}^{\prime} \rightarrow \mathbf{y}, \Delta t\right)
$$


est la probabilité de transition de l'état $\mathbf{y}^{\prime}$ vers l'état $\mathbf{y}$ durant $\Delta t$.

De plus l'éq. (1) doit satisfaire à la condition initiale

$\lim _{t \rightarrow t_{0}} P\left(\mathbf{y}, t \mid \mathbf{y}_{0}, t_{0}\right)=\delta\left(\mathbf{y}-\mathbf{y}_{0}\right)=\left\{\begin{array}{lll}1 & \text { si } & \mathbf{y} \equiv \mathbf{y}_{0} \\ 0 & \text { si } & \mathbf{y} \neq \mathbf{y}_{0}\end{array}\right.$.

Les variables aléatoires décrivant l'état $\mathbf{y}$ du réacteur sont :

$N$ : nombre total de neutrons dans le réacteur;

$C:$ nombre total de précurseurs dans le réacteur ;

$$
\theta_{\mathrm{u}}=\frac{M_{\mathrm{u}} \cdot C_{\mathrm{u}} \cdot T_{\mathrm{u}}}{q}
$$

est une quantité associée à la température $T_{\mathrm{u}}$ du combustible, dont la masse totale est $M_{\mathrm{u}}$ et la chaleur spécifique $C_{\mathrm{u}}$

$$
V=\frac{\Omega_{\mathrm{v}} \cdot \rho_{\mathrm{v}} \cdot h_{\mathrm{V}}}{q}
$$

est une quantité associée au volume $\Omega_{\mathrm{V}}$ de vapeur contenue dans le réacteur. $h_{\mathrm{v}}$ et $\rho_{\mathrm{V}}$ sont respectivement l'enthalpie de vaporisation et la densité de la vapeur ; $q$ est l'énergie moyenne libérée par fission.

Le réfrigérant (qui est aussi le modérateur et le réflecteur) est considéré comme un réservoir infini de chaleur à la température $T_{\mathrm{M}_{0}}$. A cette température constante correspond la quantité

$$
\theta_{\mathrm{M}_{\mathrm{o}}}=T_{\mathrm{M}_{\mathrm{o}}} \cdot C_{\mathrm{u}} \cdot M_{\mathrm{u}} \cdot q^{-1} \text {. }
$$

Les transitions intervenant dans l'éq. (1) sont mentionnées dans le tableau I.
Supposons $\Delta t$ suffisamment petit pour qu'un seul événement du tableau $\mathbf{I}$ conduise de l'état $\mathbf{y}^{\prime}$ à l'état $\mathbf{y}$; nous aurons [5]

$$
P\left(\mathbf{y}^{\prime} \rightarrow \mathbf{y}, \Delta t\right)=\omega_{\mathbf{y}^{\prime} \mathbf{y}} \Delta t+\left[1-\sum_{\mathbf{y}^{\prime \prime}} \omega_{\mathbf{y y}^{\prime \prime}} \Delta t\right] \delta\left(\mathbf{y}^{\prime}-\mathbf{y}\right)
$$

dans laquelle $\omega_{y^{\prime} y}$ est la probabilité par unité de temps qu'un quelconque des événements élémentaires assure la transition $\mathbf{y}^{\prime} \rightarrow \mathbf{y}\left(\omega_{\mathbf{y}^{\prime} \mathbf{y}}=0\right.$ si $\left.\mathbf{y}^{\prime} . \equiv \mathbf{y}\right)$.

Définissons de plus la probabilité de transition totale hors de l'état $\mathbf{y}^{\prime}$ durant $\Delta t$ par

$$
\Gamma_{\mathbf{y}^{\prime}} \Delta t=\sum_{\mathbf{y}^{\prime \prime}} \omega_{\mathbf{y}^{\prime} \mathbf{y}^{\prime \prime}} \Delta t .
$$

Les événements élémentaires s'excluant mutuellement, nous pouvons écrire, en relation avec le tableau I :

$$
\omega_{\mathbf{y}^{\prime} \mathbf{y}}=\sum_{i} T_{i}\left(\mathbf{y}^{\prime}\right) \cdot\left[\prod_{k} \delta\left(y_{k}^{\prime}-y_{k}+q_{i y_{k}}\right)\right] .
$$

A l'aide de ces résultats, l'éq. (1) devient :

$$
\begin{array}{r}
\frac{\partial}{\partial t} P\left(\mathbf{y}, t \mid \mathbf{y}_{0}, t_{0}\right)=\sum_{\mathbf{y}^{\prime}} P\left(\mathbf{y}^{\prime}, t \mid \mathbf{y}_{0}, t_{0}\right) \omega_{\mathbf{y}^{\prime} \mathbf{y}}- \\
-\Gamma_{\mathbf{y}} P\left(\mathbf{y}, t \mid \mathbf{y}_{0}, t_{0}\right) .
\end{array}
$$

3. Covariances et corrélations. - Les moments du $n$-ième ordre de la probabilité de transition $\omega_{\mathbf{y y}}$ sont définis par:

$$
D_{n}\left(\mathbf{y}^{n}\right)=\frac{1}{n !} \sum_{\mathbf{y}^{\prime}} \omega_{\mathbf{y y}}\left(\mathbf{y}^{\prime}-\mathbf{y}\right)^{n} .
$$

Valeurs moyennes : Moments du $1^{\mathrm{er}}$ ordre.

TABLEAU I

Evénements élémentaires dans un réacteur à eau bouillante

$i \quad$ Evénement

1. Décroissance d'un précurseur

2. Disparition stérile d'une neutron

3. Fission (libérant avec une probabilité $p\left(v_{0}, v_{1}\right) v_{0}$ neutrons et $v_{1}$ précurseurs)

4. Transfert de chaleur combustible $\rightarrow$ réfrigérant (sans formation de vapeur)

5. Transfert de chaleur combustible $\rightarrow$ réfrigérant, suivi d'apparition de vapeur

6. Transfert de chaleur réfrigérant $\rightarrow$ combustible

7. Condensation de vapeur, suivie d'un transfert de chaleur réfrigérant $\rightarrow$ combustible

8. Fuite de vapeur

9. Emission d'un neutron par une source d'intensité $S$ (neutrons/sc)

$$
\begin{gathered}
\operatorname{Taux}\left[T_{i}\right] \\
- \\
\lambda C \\
\alpha_{\mathrm{c}} N
\end{gathered}
$$$$
\alpha_{\mathrm{f}} p\left(v_{0}, v_{1}\right) N
$$$$
\alpha_{\mathrm{um}} \theta_{\mathrm{u}}
$$$$
\alpha_{\mathrm{uv}} \theta_{\mathrm{u}}
$$$$
\alpha_{\mathrm{mu}} \theta_{\mathrm{M}_{0}}
$$$$
\alpha_{\mathrm{vu}} \theta_{\mathrm{M}_{\mathbf{0}}}
$$$$
\alpha_{\mathrm{v}} V
$$$$
S
$$

Accroissement net par événement $\left[q_{i y_{k}}\right]$

\begin{tabular}{rrrr}
$N$ & $C$ & $\theta_{\mathbf{u}}$ & \multicolumn{1}{l}{} \\
- & - & - & - \\
+1 & $-\mathbf{i}$ & 0 & 0 \\
-1 & 0 & 0 & 0
\end{tabular}

$v_{0}-1$

$v_{1}$

1

0

$+1$

0

$-1$

$-1$

La signification des divers coefficients et paramètres de ce tableau est donnée en annexe. 
La valeur moyenne de $\mathbf{y}$, compte tenu des conditions initiales en $t_{0}$, sera donnée par

$$
\langle\mathbf{y}\rangle_{0}(t)=\sum_{\mathbf{y}} \mathbf{y} P\left(\mathbf{y}, t \mid \mathbf{y}_{0}, t_{0}\right) .
$$

Utilisant l'éq. (6) et la définition (7), la dérivée temporelle de (8) nous donne

$$
\frac{\mathrm{d}}{\mathrm{d} t}<\mathbf{y}>_{0}(t)=\left\langle D_{1}(\mathbf{y})>_{0} .\right.
$$

Les moments du $1^{\text {er }}$ ordre s'écrivent, en tenant compte de l'équation (5) dans la définition (7) :

$$
D_{1}(\mathbf{y})=A \cdot \mathbf{y}+\mathbf{S}
$$

avec :

$A \equiv\left[\begin{array}{crcr}\alpha_{\mathrm{f}}\left(<v_{0}>-1\right)-\alpha_{\mathrm{c}} & \lambda & 0 & 0 \\ \alpha_{\mathrm{f}}<v_{1}> & -\hat{\lambda} & 0 & 0 \\ \alpha_{\mathrm{f}} & 0 & -\left(\alpha_{\mathrm{um}}+\alpha_{\mathrm{uv}}\right) & 0 \\ 0 & 0 & \alpha_{\mathrm{uv}} & -\alpha_{\mathrm{v}}\end{array}\right]$

et

$$
\mathbf{S} \equiv\left[\begin{array}{c}
S \\
0 \\
\left(\alpha_{\mathrm{mu}}+\alpha_{\mathrm{vu}}\right) \theta_{\mathrm{M}_{0}} \\
-\alpha_{\mathrm{vu}} \theta_{\mathrm{M}_{0}}
\end{array}\right] .
$$

Nous supposerons que :

- les échanges de chaleur entre combustible et réfrigérant (liquide ou vapeur) ne dépendent que de leur différence de température; nous écrivons $\alpha_{\mathrm{um}}=\alpha_{\mathrm{mu}}$ et $\alpha_{\mathrm{uv}}=\alpha_{\mathrm{vu}}$ ce qui permet de définir $\alpha=\alpha_{\mathrm{um}}+\alpha_{\mathrm{uv}}$ et $g=\alpha_{\mathrm{uv}} / \alpha$;

- la probabilité de capture d'un neutron $\left(\alpha_{c}\right)$ dépend linéairement de la température du combustible et de la fraction de vapeur dans le réacteur ; il vient :

$$
\alpha_{c}=\alpha_{c R}-\gamma_{1}\left(\theta_{u}-\theta_{\mathrm{uR}}\right)-\gamma_{2}\left(V-V_{\mathrm{R}}\right)
$$

en supposant $\alpha_{c}$ connu pour un état de référence $y_{R}$.

Comme la réactivité est donnée par (cf. [8]) :

$$
\rho=\frac{\alpha_{\mathrm{f}}(<v>-1)-\alpha_{\mathrm{c}}}{\alpha_{\mathrm{f}}<v>}
$$

avec

$$
\langle v\rangle=\left\langle v_{0}\right\rangle+\left\langle v_{1}\right\rangle
$$

et

$$
\left\langle v_{1}\right\rangle=\beta\langle v\rangle,
$$

nous pouvons définir les coefficients de contre-réaction en réactivité suivants :

- coefficient de température du combustible :

$$
\frac{\partial \rho}{\partial T_{\mathrm{u}}}=t_{\mathrm{c}}=\frac{\gamma_{1} \cdot M_{\mathrm{u}} \cdot C_{\mathrm{u}}}{\alpha_{\mathrm{f}}<v>\cdot q}
$$

— coefficient de vide :

$$
\frac{\partial \rho}{\partial \Omega_{\mathrm{V}}}=v_{\mathrm{c}}=\frac{\gamma_{2} h_{\mathrm{V}} \rho_{\mathrm{V}}}{\alpha_{\mathrm{f}}<v>\cdot q}
$$

$\gamma_{1}$ : coefficient de température agissant sur la capture des neutrons dans le combustible (effet Doppler);

$\gamma_{2}$ : coefficient de vide agissant sur l'absorption des neutrons dans le réfrigérant (effet de densité).

Introduisons l'état $\mathbf{y}_{\mathbf{M}}$ décrivant le comportement moyen du réacteur

$$
\left(\mathbf{y}_{M}=\lim _{t_{0} \rightarrow-\infty}<\mathbf{y}>_{0}(t)\right)
$$

et écrivons

$$
\mathbf{y}=\mathbf{y}_{\mathbf{M}}+\Delta \mathbf{y} .
$$

La forme des contre-réactions nous permet d'écrire :

$$
\begin{aligned}
A & =A\left(\mathbf{y}_{\mathrm{R}}-\mathbf{y}\right)=A\left(\mathbf{y}_{\mathrm{R}}-\mathbf{y}_{\mathrm{M}}+\Delta \mathbf{y}\right) \\
& =A\left(\mathbf{y}_{\mathrm{R}}-\mathbf{y}_{\mathrm{M}}\right)+\sum_{i} A_{i} \Delta y_{i} .
\end{aligned}
$$

Alors les moments du $1^{\text {er }}$ ordre (éq. (10)) peuvent s'écrire

$$
\begin{aligned}
D_{1}(\mathbf{y})=\left[A\left(\mathbf{y}_{\mathrm{R}}-\mathbf{y}_{\mathbf{M}}\right)\right. & \left.\cdot \mathbf{y}_{\mathrm{M}}+\mathbf{S}\right]+ \\
& +\left[A\left(\mathbf{y}_{\mathrm{R}}-\mathbf{y}_{\mathbf{M}}\right)+L^{\mathrm{T}}\right] \Delta \mathbf{y}
\end{aligned}
$$

expression dans laquelle $L^{\mathrm{T}}$ est la transposée de la matrice $L$, dont les éléments sont définis par

$$
L_{i l}=\sum_{k} A_{i_{l k}} y_{\mathrm{M}, k} \text {. }
$$

L'état $\mathbf{y}_{\mathbf{M}}$ satisfait à l'équation

$$
\frac{\mathrm{d}}{\mathrm{d} t} \mathbf{y}_{\mathrm{M}}=A\left(\mathbf{y}_{\mathrm{R}}-\mathbf{y}_{\mathrm{M}}\right) \cdot \mathbf{y}_{\mathrm{M}}+\mathrm{S} .
$$

Si l'on se place dans le cas d'un réacteur stationnaire, et si l'on choisit $\mathbf{y}_{\mathbf{R}} \equiv \mathbf{y}_{\mathbf{M}}$, il viendra

$$
\frac{\mathrm{d}}{\mathrm{d} t} \mathbf{y}_{\mathrm{M}}=0
$$

et l'éq. (9) s'écrit :

$$
\frac{\mathrm{d}}{\mathrm{d} t}<\Delta \mathbf{y}>_{0}=\Lambda\left(\mathbf{y}_{\mathrm{M}}\right) \cdot\left\langle\Delta \mathbf{y}>_{0}\right.
$$

avec

$$
\Lambda\left(\mathbf{y}_{\mathrm{M}}\right)=A\left(\mathbf{y}_{\mathrm{M}}\right)+L^{\mathrm{T}}\left(\mathbf{y}_{\mathrm{M}}\right)
$$

$$
\begin{gathered}
\Lambda\left(\mathbf{y}_{\mathrm{M}}\right)=\left[\begin{array}{cccc}
\frac{\rho_{\mathrm{M}}-\beta}{\operatorname{tg}} & \lambda & \gamma_{1} N_{\mathrm{M}} & \gamma_{2} N_{\mathrm{M}} \\
\frac{\beta}{\operatorname{tg}} & -\lambda & 0 & 0 \\
\alpha_{\mathrm{f}} & 0 & -\alpha & 0 \\
0 & 0 & g \alpha & -\alpha_{\mathrm{v}}
\end{array}\right] \\
\rho_{\mathrm{M}}=\rho\left(\alpha_{\mathrm{cR}}=\alpha_{\mathrm{cM}}\right) \\
k_{\mathrm{M}}=\frac{1}{1-\rho_{\mathrm{M}}} \\
\alpha_{\mathrm{f}}<v>=\frac{k_{\mathrm{M}}}{l}=\frac{1}{\operatorname{tg}}
\end{gathered}
$$


$l=$ temps de vie des neutrons ; $t \mathrm{~g}=$ temps de génération

$k_{\mathrm{M}}=$ coefficient de multiplication effectif des neutrons.

Covariances et fonctions de corrélations. - Les fonctions de covariances sont définies par

$$
<y_{k} \cdot y_{l}>_{0}(t)=\sum_{\mathbf{y}} y_{k} y_{l} P\left(\mathbf{y}, t \mid y_{0}, t_{0}\right)
$$

ou, sous forme matricielle

$$
<y^{2}>_{0}(t)=\sum_{\mathbf{y}} y^{2} P\left(\mathbf{y}, t \mid \mathbf{y}_{0}, t_{0}\right) \text {. }
$$

En dérivant l'éq. (18) par rapport au temps, il vient $\begin{aligned} \frac{\mathrm{d}}{\mathrm{d} t}<y_{k} \cdot y_{l}>_{0}=2<D_{2}\left(y_{k} y_{l}\right)> & +<y_{k} D_{1}\left(y_{l}\right)> \\ & +<y_{l} D_{1}\left(y_{k}\right)>\end{aligned}$

ou

$$
\frac{\mathrm{d}}{\mathrm{d} t}<y^{2}>_{0}=2<D_{2}\left(y^{2}\right)>+2<y \cdot D_{1}(y)>.
$$

En utilisant l'état $\mathbf{y}_{\mathbf{M}}$ et les résultats précédemment obtenus pour $D_{1}(\mathbf{y})$, nous obtenons les fonctions de covariances des variables centrées $\Delta \mathbf{y}=\mathbf{y}-\mathbf{y}_{\mathbf{M}}$ :

$$
\begin{gathered}
\frac{\mathrm{d}}{\mathrm{d} t}<\Delta y^{2}>_{0} \cong 2 D_{2}\left(y_{\mathrm{M}}\right)+<\Delta y^{2}>_{0} \Lambda^{\mathrm{T}}\left(y_{\mathrm{M}}\right)+ \\
+\Lambda\left(y_{\mathrm{M}}\right)<\Delta y^{2}>_{0} .
\end{gathered}
$$

Introduisons alors la probabilité jointe $P\left(\mathbf{y}_{1}, t_{1} ; \mathbf{y}_{2}, t_{2}\right)$ d'existence de l'état $\mathbf{y}_{1}$ au temps $t_{1}$ et de l'état $\mathbf{y}_{2}$ au temps $t_{2}\left(t_{1} \leqslant t_{2}\right)$.
Nous pouvons définir les fonctions de corrélation :

$$
\begin{gathered}
\left\langle y_{1_{k}} y_{2_{l}}\right\rangle\left(t_{1}, t_{2}\right)=\sum_{\mathbf{y}_{1_{k}}, \mathbf{y}_{2_{k}}} y_{1_{k}} y_{2_{l}} P\left(\mathbf{y}_{1}, t_{1} ; \mathbf{y}_{2}, t_{2}\right) \text {, } \\
t_{1} \leqslant t_{2}
\end{gathered}
$$

Ainsi, comme le fait par exemple A. Dalfès [10] en utilisant l'équation de Fokker-Planck pour les probabilités jointes, nous obtenons

$$
\begin{aligned}
\frac{\partial}{\partial t_{2}}<y_{1_{k}} y_{2_{l}}>\left(t_{1}, t_{2}\right)=<y_{1_{k}} . D_{1}\left(y_{2_{l}}\right) & > \\
t_{1} & \leqslant t_{2}
\end{aligned}
$$

ou, pour les variables centrées (sous forme matricielle) :

$$
\begin{aligned}
\frac{\partial}{\partial t_{2}}<\Delta \mathbf{y}_{1} \cdot \Delta \mathbf{y}_{2}>\left(t_{1}, t_{2}\right)=<\Delta \mathbf{y}_{1} \Delta \mathbf{y}_{2}>\Lambda^{\mathrm{T}}\left(\mathbf{y}_{M}\right) \\
t_{1} \leqslant t_{2} .
\end{aligned}
$$

4. Densité spectrale de bruit. - Dans le cas d'un réacteur stationnaire critique (donc $\rho_{\mathrm{M}}=0, S=0$ ), et utilisant les résultats précédents (éq. (20) et (23)), nous obtenons facilement (ainsi que l'on peut le vérifier dans [5], [7] ou [8]) la transformée de Fourier des fonctions de corrélations des variables centrées, ce qui donne la matrice des densités spectrales énergétiques de bruit :

$$
\begin{array}{rl}
S(f)=\left[i 2 \pi f I-\Lambda\left(\mathbf{y}_{M}\right)\right]^{-1} & 2 D_{2}\left(\mathbf{y}_{M}^{2}\right) \\
& {\left[-i 2 \pi f I-\Lambda^{\mathrm{T}}\left(\mathbf{y}_{M}\right)\right]^{-1}}
\end{array}
$$

dans laquelle $\Lambda\left(\mathbf{y}_{\mathbf{M}}\right)$ est définie par l'éq. (16), $I \equiv$ matrice unité, et après calculs :

$$
2 D_{2}\left(\mathbf{y}_{\mathbf{M}}^{2}\right)=\left[\begin{array}{cccc}
\alpha_{\mathrm{t}} N_{\mathrm{M}} & * & * & * \\
\alpha_{l} N_{\mathrm{M}} & \alpha_{\mathrm{m}} N_{\mathrm{M}} & * & * \\
\alpha_{\mathrm{f}}\left(<v_{0}>-1\right) N_{\mathrm{M}} & \alpha_{\mathrm{f}} \beta<v>N_{\mathrm{M}} & 2 \alpha_{\mathrm{f}} N_{\mathrm{M}}+2 \alpha \theta_{\mathrm{M}_{\mathrm{o}}} & * \\
0 & 0 & -g \alpha_{\mathrm{f}} N_{\mathrm{M}}-2 g \alpha \theta_{\mathrm{M}_{0}} & g \alpha_{\mathrm{f}} N_{\mathrm{M}}+2 g \alpha \theta_{\mathrm{M}_{0}}
\end{array}\right]
$$

avec

$$
\begin{aligned}
& \alpha_{\mathrm{t}}=\alpha_{\mathrm{f}}<v_{0}\left(v_{0}-1\right)>+2 \alpha_{\mathrm{f}}<v_{1}>, \\
& \alpha_{\mathrm{m}}=\alpha_{\mathrm{f}}<v_{1}\left(v_{1}+1\right)>, \\
& \alpha_{1}=\alpha_{\mathrm{f}}<v_{1}\left(v_{0}-1\right)>-\alpha_{\mathrm{f}}<v_{1}>,
\end{aligned}
$$

la matrice $2 D_{2}\left(\mathbf{y}_{M}^{2}\right)$ étant symétrique, comme l'indique le signe *.

La densité spectrale énergétique du bruit neutronique (DSN) s'obtient en calculant le premier élément diagonal de $S(f)$. Toutefois, l'expression exacte est trop compliquée pour être reproduite ici, et nous en donnerons une forme simplifiée dans la suite.

5. Fonction de transfert. - Nous avions obtenu, pour les équations aux valeurs moyennes, la relation (9)

$$
\frac{\mathrm{d}}{\mathrm{d} t}<\mathrm{y}>_{0}(t)=\left\langle D_{1}(\mathrm{y})\right\rangle_{0}
$$

en négligeant les covariances, et en se plaçant dans le

cas d'un réacteur stationnaire (caractérisé par $\mathbf{y}_{\mathbf{M}}$ ) ce système permet de calculer, après transformation de Laplace, la réponse $\Delta N(s)$ à un saut de réactivité $\Delta k$. Il vient après calcul

$$
T(s)=\frac{\frac{\Delta N(s)}{N_{\mathrm{M}}}}{\frac{\Delta k}{k_{\mathrm{M}}}}=\frac{T_{0}(s)}{1-T_{0}(s) \cdot T_{\mathrm{F}}(s)}
$$

avec

$$
T_{0}(s)=\frac{1}{s\left(l_{M}+\frac{\beta k_{M}}{s+\lambda}\right)+1-k_{M}}
$$

fonction de transfert en boucle ouverte

$$
T_{\mathrm{F}}(s)=l_{\mathrm{M}} N_{\mathrm{M}}\left[\gamma_{1} \frac{\alpha_{\mathrm{f}}}{s+\alpha}+\gamma_{2} \frac{\alpha_{\mathrm{f}}}{s+\alpha} \frac{\alpha g}{s+\alpha_{\mathrm{v}}}\right]
$$

fonction de transfert de contre-réaction. 
Nous pouvons ainsi facilement calculer le module au carré de la fonction de transfert (FT) qui s'obtient $\operatorname{par}|T(i 2 \pi f)|^{2}$.

La fonction de transfert $T(s)$ obtenue après calculs correspond au schéma bouclé suivant :

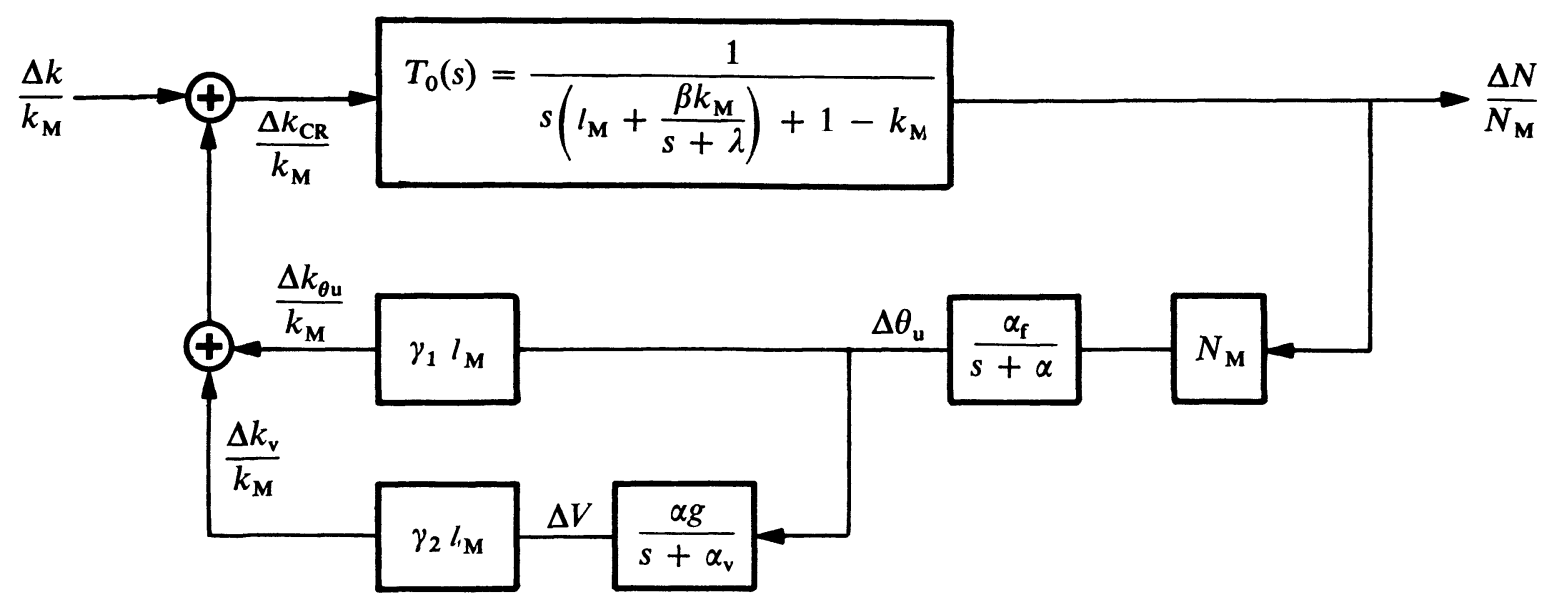

$T_{0}(s)$ est la fonction de transfert reliant les fluctuations de réactivité aux fluctuations de la population neutronique du réacteur.

Deux sources de bruit de réactivité sont mises en évidence par ce schéma, toutes deux proportionnelles à la puissance $\left(N_{\mathrm{M}}\right)$ du réacteur :

- Un bruit de température $\left(\Delta k_{\theta_{\mathrm{u}}}\right)$ dont l'intensité dépend de $\gamma_{1}(\sim$ coefficient Doppler) et relié aux fluctuations de puissance par la fonction de transfert puissance-température du combustible

$$
\frac{\alpha_{\mathrm{f}}}{s+\alpha} \text {. }
$$

- Un bruit d'ébullition $\left(\Delta k_{\mathrm{v}}\right)$, d'intensité proportionnelle au coefficient de vide $\left(\sim \gamma_{2}\right)$ et relié aux fluctuations de température du combustible par la fonction de transfert température du combustible-vide

$$
\frac{\alpha g}{s+\alpha_{v}} \text {. }
$$

Remarquons que dans les réacteurs à eau bouillante, nous avons généralement $\left|\gamma_{1}\right| \ll\left|\gamma_{2}\right|$, et par conséquent $\Delta k_{\theta_{\mathrm{u}}}$ peut être négligé devant $\Delta k_{\mathrm{v}}$.

6. Bruit d'entrée. - Le bruit d'entrée $I$ du réacteur est défini par la relation

$$
S(f)=|T(f)|^{2} I(f)
$$

ou par

$$
I(\omega)=\frac{S(\omega)}{|T(i \omega)|^{2}} \quad(\omega=2 \pi f) .
$$

7. Formes analytiques approximées. - Compte tenu des valeurs numériques caractéristiques d'un réacteur stationnaire, critique à eau bouillante (cf. [3]), et reproduite dans le tableau II, les fonctions calculées ci-dessus (DSN, FT, BE) peuvent être approximées par les formes analytiques suivantes (avec $\omega=2 \pi f$; $\alpha_{\mathrm{p}}=-\beta / l$ ): (les formes analytiques exactes, trop compliquées, ne sont pas reproduites ici, mais leurs caractéristiques seront présentées plus loin).

\section{TABLEAU II}

\section{Exemple de valeurs numériques des constantes physiques d'un réacteur à eau bouillante}

(Les valeurs sont exprimées dans le système MKSA)

$\begin{array}{rlrlrl}\rho & =0 & \beta & =700 \times 10^{-5} & \lambda & =5 \times 10^{-2} \mathrm{~s}^{-1} \\ N_{\mathrm{M}} & \left.=2 \times 10^{15} \text { (ce qui correspond à une puissance thermique de } 1000 \mathrm{MW}\right) & & \\ <v> & =2,5 & l=3 \times 10^{-5} \mathrm{~s}^{-1} & & t_{\mathrm{c}}=-2 \times 10^{-5} / \mathrm{K} \\ q & =3,2 \times 10^{-11} \mathrm{~J} & & C_{\mathrm{u}}=330 \mathrm{~J} / \mathrm{kg} \mathrm{K} \\ T_{\mathrm{M}} & =565 \mathrm{~K} & M_{\mathrm{u}}=75750 \mathrm{~kg} & g, 77 \\ \alpha_{\mathrm{v}} & =2,44 \mathrm{~s}^{-1} & \alpha & =0,2 \mathrm{~s}^{-1} & & \rho_{\mathrm{V}}=40 \mathrm{~kg} / \mathrm{m}^{3} \\ v_{\mathrm{c}} & =-1,85 \mathrm{~m}^{-3} & h_{\mathrm{V}}=1,46 \times 10^{6} \mathrm{~J} / \mathrm{kg} & \alpha_{\mathrm{t}}=7,3 \times 10^{4} \mathrm{~s}^{-1}\end{array}$


Densité spectrale énergétique du bruit neutronique (DSN).

$$
S_{\mathrm{NN}}^{\mathrm{A}}(\omega)=N_{\mathrm{M}} \alpha_{\mathrm{t}} \frac{\left[\omega^{2}+\lambda^{2}\right]\left[\omega^{2}+\alpha^{2} \frac{\alpha_{\mathrm{f}} N_{\mathrm{M}}+\alpha \theta_{\mathrm{Mo}}(1-g)}{\alpha_{\mathrm{f}} N_{\mathrm{M}}+\alpha \theta_{\mathrm{M}_{0}}}\right]\left[\omega^{2}+\frac{2 N_{\mathrm{M}}}{\alpha_{\mathrm{t}}} g \gamma_{2}^{2}\left(\alpha_{\mathrm{f}} N_{\mathrm{M}}+\alpha \theta_{\mathrm{M}_{0}}\right)\right]}{\left[\omega^{2}+\lambda^{2}\right]\left[\omega^{2}-\frac{\alpha_{\mathrm{f}} N_{\mathrm{M}} \alpha g \gamma_{2}}{\alpha_{\mathrm{p}}}\right]^{2}\left[\omega^{2}+\alpha_{\mathrm{p}}^{2}\right]+2\left(\lambda \alpha_{\mathrm{f}} N_{\mathrm{M}}\right)^{2} \alpha_{\mathrm{v}} \gamma_{1} \alpha g \gamma_{2}}
$$

Fonction de transfert (FT).

$$
\left|T^{\mathrm{A}}(i \omega)\right|^{2}=\frac{1}{l_{\mathrm{M}}^{2}} \frac{\left(\omega^{2}+\alpha^{2}\right)\left(\omega^{2}+\alpha_{\mathrm{v}}^{2}\right)\left(\omega^{2}+\lambda^{2}\right)}{\left[\omega^{2}+\lambda^{2}\right]\left[\omega^{2}-\frac{\alpha_{\mathrm{f}} N_{\mathrm{M}} \alpha g \gamma_{2}}{\alpha_{\mathrm{p}}}\right]^{2}\left[\omega^{2}+\alpha_{\mathrm{p}}^{2}\right]+2\left(\lambda \alpha_{\mathrm{f}} N_{\mathrm{M}}\right)^{2} \alpha_{\mathrm{v}} \gamma_{1} \alpha g \gamma_{2}} .
$$

Nous remarquons que les dénominateurs de $S_{\mathrm{NN}}^{\mathrm{A}}(\omega)$ et de $\left|T^{\mathrm{A}}(i \omega)\right|^{2}$ sont identiques. Cela s'explique par le fait que les dénominateurs des fonctions exactes $S_{\mathrm{NN}}(\omega)$ et $|T(i \omega)|^{2}$ sont eux-mêmes identiques.

Bruit d'entrée (BE).

$$
I^{A}(\omega)=N_{M} l_{M}^{2} \alpha_{t} \frac{\left[\omega^{2}+\alpha^{2} \frac{\alpha_{\mathrm{f}} N_{\mathrm{M}}+\alpha \theta_{\mathrm{Mo}}(1-g)}{\alpha_{\mathrm{f}} N_{\mathrm{M}}+\alpha \theta_{\mathrm{M}_{0}}}\right]\left[\omega^{2}+\frac{2 N_{\mathrm{M}}}{\alpha_{\mathrm{t}}} g \gamma_{2}^{2}\left(\alpha_{\mathrm{f}} N_{\mathrm{M}}+\alpha \theta_{\mathrm{M}_{0}}\right)\right]}{\left[\omega^{2}+\alpha^{2}\right]\left[\omega^{2}+\alpha_{\mathrm{v}}^{2}\right]} .
$$

8. Analyses paramétriques. - Puissance variable. - Si la puissance du réacteur augmente, on peut constater :

SUR LA DSN (Fig. 1).

- Une baisse relative de l'amplitude des fluctuations basses fréquences $\left(\omega<\lambda, N_{\mathrm{M}}<2 \times 10^{14}\right)$.

- L'apparition simultanée d'une résonance située dans une plage de fréquences intermédiaires $\left(10^{-2} \mathrm{~s}^{-1}<\omega<10^{1} \mathrm{~s}^{-1}\right.$, dès que $\left.N_{\mathrm{M}}>2 \times 10^{12}\right)$.

- Une augmentation générale de l'amplitude de la DSN, proportionnelle à $N_{\mathrm{M}}$ pour $\omega>10^{1} \mathrm{~s}^{-1}$ et $N_{\mathrm{M}}<2 \times 10^{17}$ et également proportionnelle à $N_{\mathrm{M}}$ pour $\omega<\lambda, N_{\mathrm{M}}>2 \times 10^{16}$.

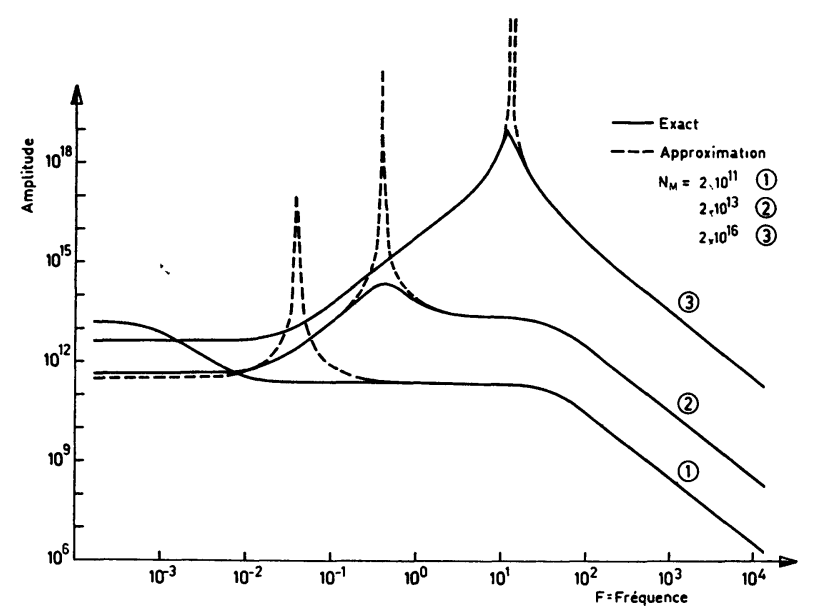

FIG. 1. - Densité spectrale énergétique du bruit neutronique (DSN). Puissance variable.

\section{SUR LA FT (Fig. 2).}

- Une baisse relative de l'amplitude de la FT aux basses fréquences $(\omega<\lambda)$ qui est proportionnelle à $N_{\mathrm{M}}^{2}$.
- L'apparition d'une résonance identique à celle observée sur la DSN.

- Un comportement indépendant de $N_{\mathrm{M}}$ aux fréquences élevées, c'est-à-dire supérieures à la fréquence de résonance.

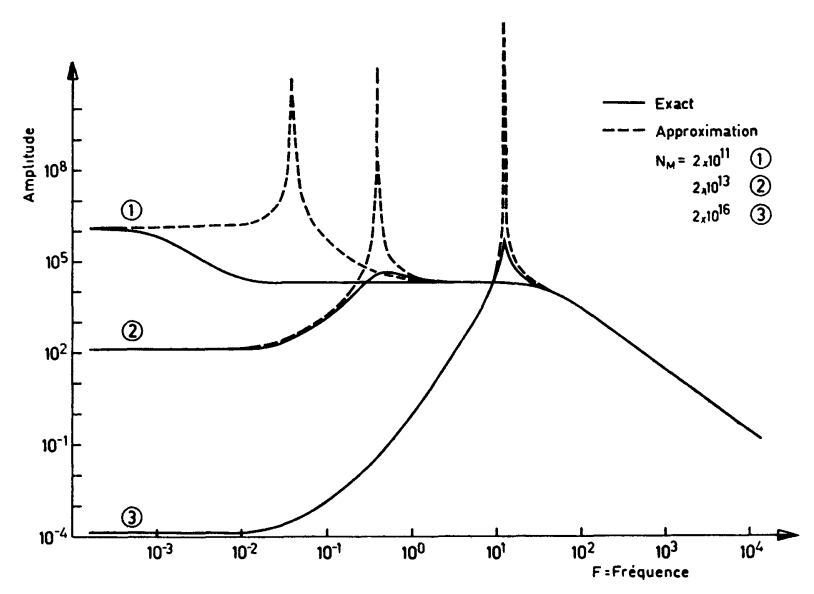

FIG. 2. - Fonction de transfert (FT). Puissance variable.

\section{SUR LE BE (Fig. 3).}

- Un comportement indépendant de la fréquence pour de faibles puissances $\left(N_{\mathrm{M}}<2 \times 10^{12}\right)$, ce qui indique bien que le bruit d'entrée est blanc dans ce cas, donc que les effets des contre-réactions sont négligeables (shot noise).

- Une augmentation relative du niveau de bruit aux basses fréquences caractéristiques des contreréactions (flicker noise), ainsi qu'une augmentation générale de l'amplitude de bruit. On constate que le flicker noise croît plus vite avec la puissance que le shot noise (cf. [4]). 


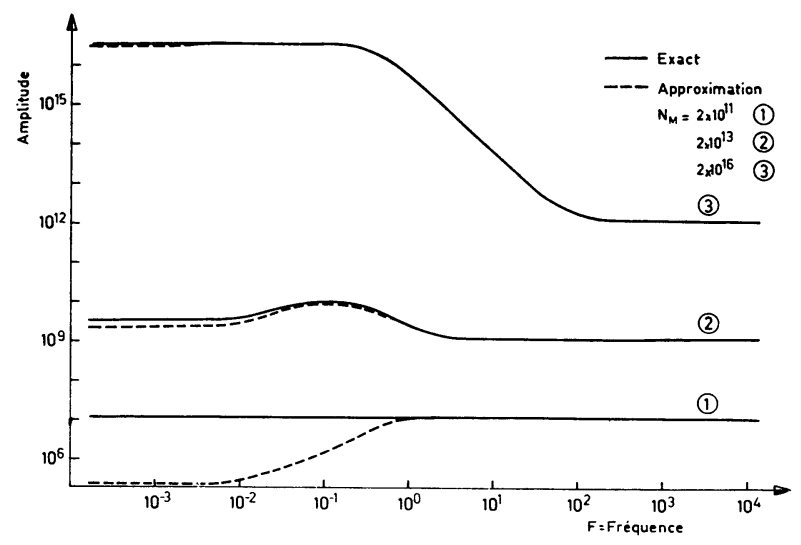

FIG. 3. - Bruit à l'entrée (BE). Puissance variable.

CoEfFicient DE VIDE VARIABLE. - Si le coefficient de vide $\left(\left|v_{\mathrm{c}}\right|\right)$ croît, on constate principalement :

\section{SUR LA DSN (Fig. 4).}

- Une diminution relative de l'amplitude des fluctuations basses fréquences $(\omega<\lambda)$ (se stabilisant pour $\left.v_{\mathrm{c}} \cong-2 \times 10^{-1} \mathrm{~m}^{-3}\left(-2 \times 10^{-2} \mathrm{pcm} / \mathrm{cm}^{3}\right)\right)$, et une augmentation de l'importance de la résonance dans l'allure générale de la DSN ; ceci provient du déplacement du pic vers les fréquences élevées si $\left|v_{\mathrm{c}}\right|$ croît, en entraînant la disparition du plateau situé à droite du pic.

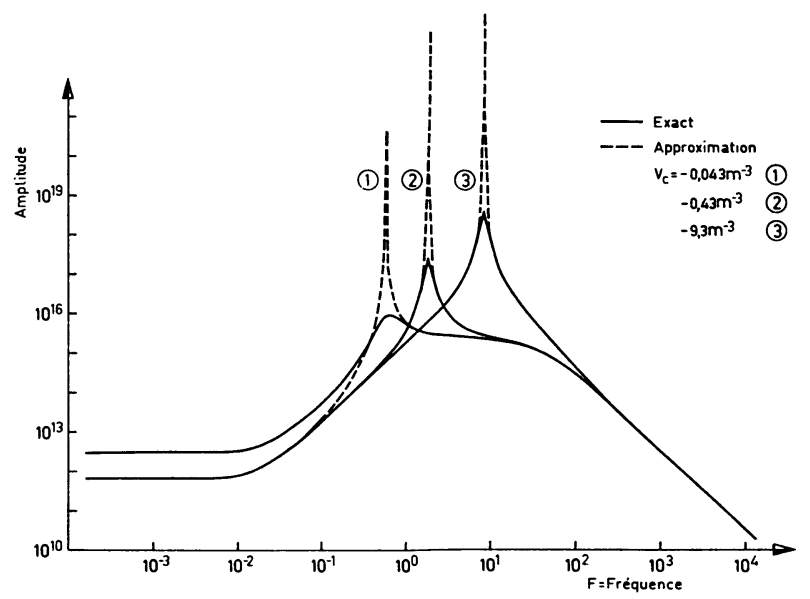

Fig. 4. - Densité spectrale énergétique du bruit neutronique (DSN). Coefficient de vide variable.

\section{SUR LA FT (Fig. 5).}

- Des effets identiques à ceux observés sur la DSN, mis à part la non-stabilisation de la diminution des amplitudes de la FT aux pulsations inférieures à $\lambda$.

\section{SUR LE BE (Fig. 6).}

- Une augmentation du niveau de bruit aux basses fréquences (découlant naturellement des remarques précédentes), alors qu'il est indépendant de la valeur de $v_{\mathrm{c}}$ pour les fréquences élevées $\left(\omega>10^{3} \mathrm{~Hz}\right)$.

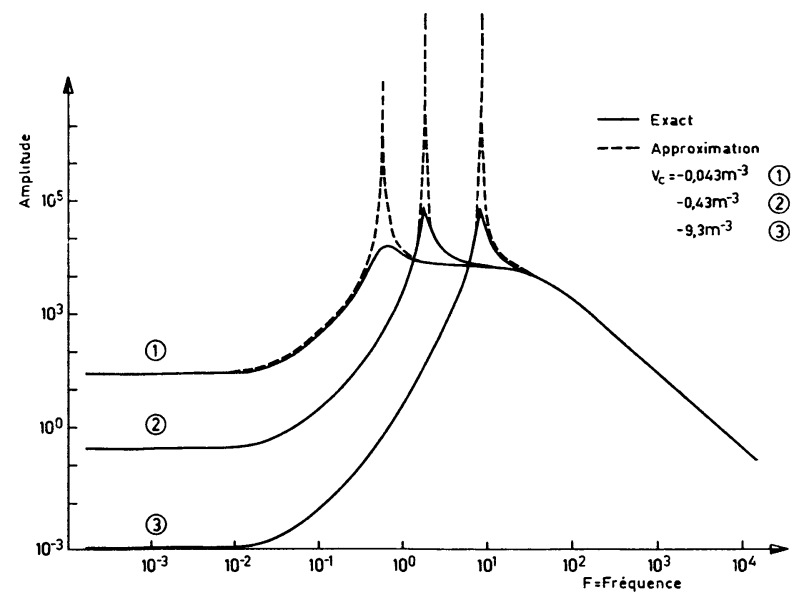

Fig. 5. - Fonction de transfert (FT). Coefficient de vide variable.

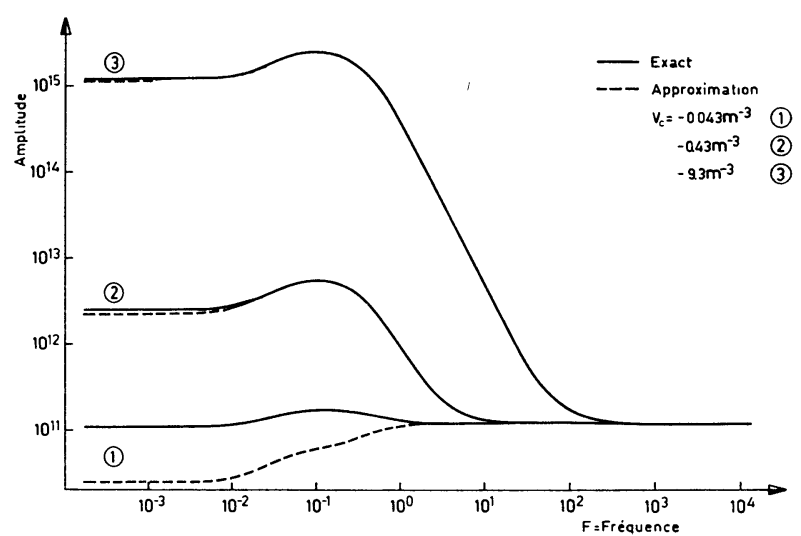

Fig. 6. - Bruit à l'entrée (BE). Coefficient de vide variable.

\section{Remarques finales.}

- Ainsi que nous pouvons le constater sur les figures, les fonctions analytiques simplifiées proposées constituent une approximation satisfaisante des fonctions exactes pour les plages de valeurs suivantes : $10^{14}<N_{\mathrm{M}}<10^{17}$ et $-4 \mathrm{~m}^{-3}<v_{\mathrm{c}}<-0,2 \mathrm{~m}^{-3}$.

- En se basant sur la forme approximée de la DSN et de la FT, on constate que celles-ci présentent une résonance pour une pulsation voisine de

$$
\omega_{0}=\left(-\frac{\alpha_{\mathrm{f}} N_{\mathrm{M}} \alpha g \gamma_{2}}{\alpha_{\mathrm{p}}}\right)^{1 / 2}
$$

soit

$$
\sim 4,2 \times 10^{-7}\left(-N_{\mathrm{M}} \cdot v_{\mathrm{c}}\right)^{1 / 2} s^{-1}
$$

(en tenant compte des valeurs numériques du tableau II), ce qui est bien ce que l'on observe. De plus, on remarque que le pic se déplace bien vers les fréquences croissantes comme $\left(-N_{\mathrm{M}} v_{\mathrm{c}}\right)^{1 / 2}$.

- Les observations faites, à savoir :

- diminution de l'amplitude relative des fluctua- 
tions pour $\omega<\lambda$, tant sur la DSN que sur la FT avec $N_{\mathrm{M}}$ ou $\left|v_{\mathrm{c}}\right|$ croissant,

- l'augmentation plus rapide du flicker noise que du shot noise pour les mêmes pulsations, avec également $N_{\mathrm{M}}$ et $\left|v_{\mathrm{c}}\right|$ croissant,

- l'existence d'une fréquence de coupure

$$
f_{\mathrm{c}}=+\frac{\beta}{2 \pi l}\left(\omega_{\mathrm{c}}=\frac{\beta}{l}=-\alpha_{\mathrm{p}}\right)
$$

sur la DSN et FT, identique à celle observée pour les réacteurs de puissance nulle,

sont en accord avec celles faites par différents auteurs, par exemple K. Saito [4], R. Isnard [8] et D. R. Harris [2].

Actuellement une étude est en cours ayant pour but de déterminer les conditions nécessaires à la réalisation d'une expérience simulant l'ébullition naturelle dans un réacteur à uranium enrichi-eau légère (réacteur CROCUS de l'EPF-Lausanne, puissance maximum $100 \mathrm{~W})$. Cette expérience a pour but de vérifier la validité des hypothèses et du modèle présenté ici.

\section{ANNEXE}

Nomenclature relative au tableau I. - Les coefficients et paramètres introduits dans le tableau I ont la signification suivante :

$\alpha_{\mathrm{f}}$ : probabilité par unité de temps et par neutron pour qu'un neutron crée une fission.

$\alpha_{c}$ : probabilité par unité de temps et par neutron pour que ce dernier disparaisse par capture dans le réacteur ou par fuite hors de celui-ci. $\langle v\rangle=\left\langle v_{0}\right\rangle+\left\langle v_{1}\right\rangle$ : nombre moyen de neutrons prompts $\left(<v_{0}>\right)$ et retardés $\left(<v_{1}>\right)$ émis par fission (les moyennes sont calculées avec la distribution $\left.p\left(v_{0}, v_{1}\right)\right)$.

$\beta$ : fraction effective des neutrons retardés $\left.\left(=\left\langle v_{1}\right\rangle /<v\right\rangle\right)$.

$\lambda$ : probabilité par unité de temps et par précurseur pour qu'un précurseur se désintègre, en émettant un neutron (dit retardé).

$\alpha_{\text {um }}$ : probabilité par unité de temps et par unité d'énergie interne du combustible pour que cette dernière décroisse d'une unité par transfert au réfrigérant, sans génération de vapeur.

$\alpha_{\mathrm{mu}}$ : probabilité par unité de temps et par unité d'énergie interne du modérateur pour que la quantité d'énergie interne du combustible croisse d'une unité par transfert du réfrigérant.

$\alpha_{\mathrm{uv}}$ : probabilité par unité de temps et par unité d'énergie interne du combustible pour que cette dernière décroisse d'une unité par transfert au réfrigérant, immédiatement suivi de génération de vapeur.

$\alpha_{\mathrm{vu}}$ : probabilité par unité de temps et par unité d'énergie interne du modérateur pour que le nombre d'unités d'enthalpie de vapeur décroisse d'une unité par condensation et transfert au combustible.

$\alpha_{\mathrm{v}}^{*}$ : probabilité par unité de temps et par unité d'enthalpie de vapeur pour que cette dernière décroisse d'une unité par fuite de vapeur hors du réacteur.

Pour toutes ces grandeurs, l'indice $\mathrm{R}$ ou $\mathrm{M}$ indique qu'elles seront supposées connues pour un état de référence $\mathbf{y}_{\mathbf{R}}$ du réacteur ou pour l'état $\mathbf{y}_{\mathbf{M}}$ décrivant le comportement moyen de ce dernier.

\section{Bibliographie}

[1] ThIE, J. A., Neutron Noise an AEC Monograph, (Rowman and Littlefield Inc., New York) 1963.

[2] Harris, D. R., Stochastic Fluctuations in a Power Reactor WAPD-TM-190 (Bettis Atomis Power Laboratory) 1958 .

[3] Pluta, R. P., An Analysis of Fluctuations in a Boiling Water Reactor by Methods of Stochastic Processes, APED-4071, General Electric (1962).

[4] Saito, K., Input-Noise Source of At Power Reactors with Temperature Feed-back Effect, Nucl. Sc. Eng. 37 (1969) 380.

[5] Morishima, N., Theory of Stochastic Fluctuations in a Power Reactor, J. Nucl. Sc. Technol. 10 (1973) 1.

[6] Kosaly, G., Maroti, L., Mesko, L., A simple Space Dependent Theory of the Neutron Noise in a Boiling
Water Reactor, Ann. Nucl. Energy, 2 (1975), n 2-5, p. 315.

[7] Lax, M., Classical Noise IV : Langevin Methods, Rev. Mod. Phys. 38 (1966) 541.

[8] ISNARD, R., Processus aléatoires dans un réacteur nucléaire compte tenu des effets de la température du combustible. $1^{\mathrm{re}}$ partie : Etablissement du modèle. $2^{\mathrm{e}}$ partie : Détermination expérimentale des paramètres du réacteur, Revue Phys. Appl. 8 (1973) 1.

[9] LAX, M, Fluctuation from the Non-Equilibrium Steady State, Rev. Mod. Phys. 32 (1960).

[10] Dalfes, A., The Correlation Function and Power Spectral Density of Nuclear Reactors, Nukleonik 6, Heft 2 (1964). 